Témoigner Témoigner. Entre histoire et mémoire

Getuigen Revue pluridisciplinaire de la Fondation Auschwitz

$123 \mid 2016$

Traduire le témoignage

\title{
Bridge of spies / Le pont des espions : héros de l'ombre
}

Bridge of Spies: Helden in de schaduw

\section{Erik Machielsen}

\section{Q OpenEdition}

\section{Journals}

\section{Édition électronique}

URL : https://journals.openedition.org/temoigner/5048

DOI : $10.4000 /$ temoigner. 5048

ISSN : 2506-6390

\section{Éditeur :}

Éditions du Centre d'études et de documentation Mémoire d'Auschwitz, Éditions Kimé

\section{Édition imprimée}

Date de publication : 1 octobre 2016

Pagination : 13-16

ISBN : 987 2-9600926-4-6

ISSN : 2037-4183

Référence électronique

Erik Machielsen, "Bridge of spies / Le pont des espions : héros de l'ombre », Témoigner. Entre histoire et mémoire [En ligne], 123 | 2016, mis en ligne le 02 novembre 2021, consulté le 04 novembre 2021. URL: http://journals.openedition.org/temoigner/5048 ; DOI : https://doi.org/10.4000/temoigner.5048 


\section{BRIDGE OF SPIES / LE PONT DES ESPIONS HEROS DE L'OMBRE}

CINEMA Tiens! Encore un de ces films qui traitent de la guerre froide. On commence à en connaitre l'air. L'air? Oui. Mais pas la chanson. Car quand on cumule à un même générique Steven Spielberg et Tom Hanks, ça vaut peut-être la peine d'aller y regarder de plus près.
Après 28 films à son actif, Steven Spielberg n'a rien perdu de son souffle. Bridge of Spies témoigne plus que jamais de son penchant pour les héros de l'ombre, ces messieurs et mesdames Tout-le-Monde pour qui les moteurs sont l'intégrité et la conscience.

\section{UN GARS NORMAL}

Toutes proportions et contexte gardés, son Schindler est ici James Britt Donovan, un Américain ordinaire, fidèle à sa femme, un père de famille aimant, baignant dans sa routine de bon citoyen. Un gars sans aspérités que l'on remarque peu, qui se fond dans la foule. Un homme discret qui va pourtant se montrer redoutablement efficace.

C'est que sans son existence, la face du monde eut probablement été changée. Ceux qui étaient adolescents au début des années 1960 s'en souviennent peut-être. C'était l'époque des deux K (Kennedy et Kroutchev).

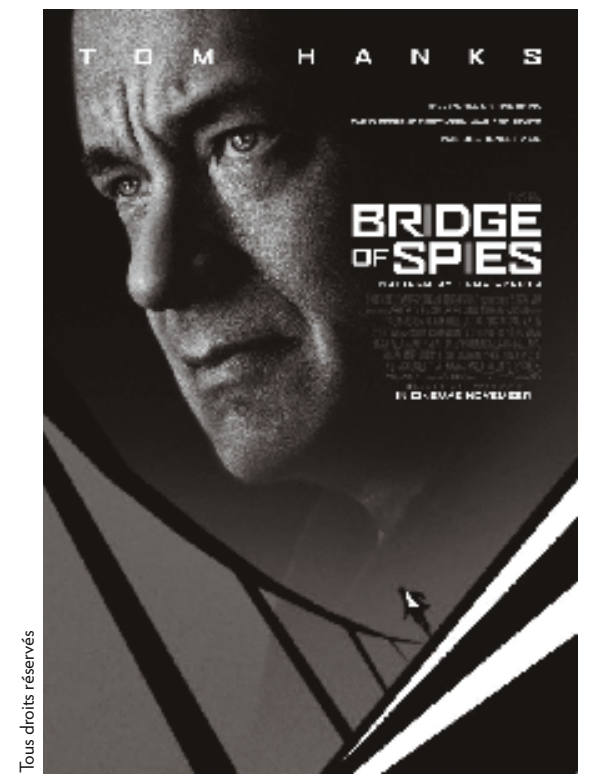

La télévision et les journaux faisaient leurs unes avec un incident dont ils redoutaient qu'il allume la mèche d'une guerre mondiale entre les deux grandes puissances d'alors.

\section{ABEL ? FISHER ?}

On est à ce moment en pleine course aux armements entre les États-Unis et l'URSS. Chacun détient l'arme nucléaire et il est indispensable de connaître l'état d'avancement et les méthodes de travail de l'un si l'autre veut garder la main. D’où le rôle essentiel joué par les services d'espionnage. On trouve, côté russe, un certain William Fisher. Lequel emprunte plusieurs noms, dont celui de Rudolf Abel sous lequel son procès se déroula. Spielberg, parce qu'il précise que le film est «inspiré » de faits réels - ce qui lui permet certaines diversions et raccourcis - et par souci de clarté, s'en est tenu à celui-là.

Fin 1948, donc, trompant les services d'immigration et après être passé par le Canada, Fisher s'introduit aux USA. Sa mission? Fournir des renseignements sur le programme nucléaire des États-Unis et organiser un réseau de radio communication avec Moscou. Il s’installe alors à Brooklyn, usurpant l'identité d'un Américain décédé : Emil Goldfus. Sa couverture : artiste peintre.

\section{AVION - ESPION}

Côté Amérique, quelques années plus tard, la CIA mandate six pilotes de U2 équipés de caméras pour survoler et filmer, en violant son espace aérien, le territoire soviétique. Un des avions est abattu. Son pilote, Francis Gary Powers, a le temps de s'en extraire et de sauter en parachute. Arrivé à terre, un comité d'accueil l'attend. Direction : la prison de Vladmir. Motif : espionnage. •.• 
.. Problème pour la CIA : Gary Power ne croque pas sa pilule de cyanure. Ce qui aurait garanti qu' il n'en vienne áfen Défense silétaitsoumisà la question. Cée lui fut d'ailleurs repróné, dé clairement indiqué dans le film.

Le Russe aux USA, l'Américain en LSS. Un jour ces deux homain URSS. Un jour ces deux hommes se de Glienicke qui relie Berlin a Pont

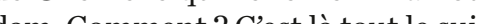
de Bridge of Spies.

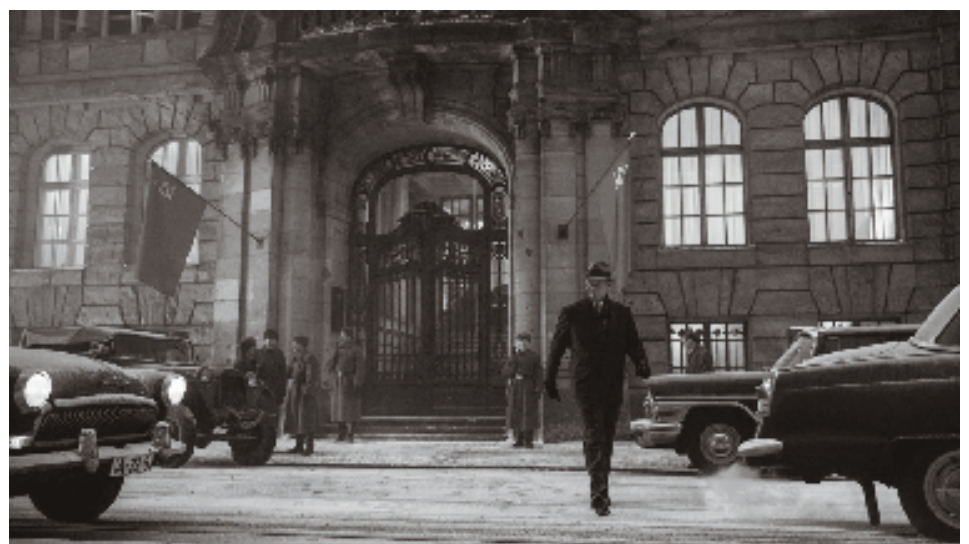

BRIDGE OF SPIES, SCĖNE 1, ACTION

1957 : Quoi de plus inoffensif qu’un homme d’âge moyen qui peint méticuleusement les ponts de Broo-

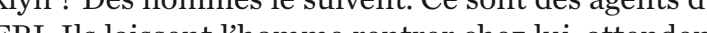
BI. Is laissent Thomme rentrer chez lui, attendent unpeu. Puis pénètrent en force dans sonpetit apparte-

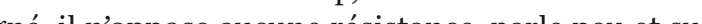
docil in

Étonnammentcalne toujous poli, cethomane, Étonnamment calme et toujours poli, cet homm Rudolf Abel, ne reconnait pas ce dont on le soupçonn trefuse de coopérer avec le FBI en échange de reneustrair a un soustraire à un proces. Et comme on ne badine pas avec l'espionnage en Amérique (que l'on se rappelle devrait le conduire tout doit a devrait le conduire tout droit à la chaise electrique.

Mais pour que les Américans ne soient pas pris en deffaut en pratiquant les méthodes staliniennes quilis eprochent à leur adversaire, lls se doivent de faire les Áses - dans la forne tout au noins - dans les règles. Acet effet, James B. Donovan, un avocat spécialisé dan ces assurances, mais quieut loccasion de travailler avec a CIA durant la guerre est sollicité. Mais voilà : pour Donovan, le Droit constitutionnel dort être applique à tion par y conidoiries, d'épronner. er la mort à son client.

WOULD IT HELP ?

Donovan rencontre Abel. Le courant passe entre les deux. Ce qui les lie n'est pas et ne sera jamais de la complicité. Mais die Ael ne se démonte pas, Donovan l'interroge : «Vous n’avez pas peur ?» Et Abel de lui répondre : «Would it help ?» (je vou écris en anglais, ça sonne tellement mieux !) Une phrase qu'il répétera à plusieurs reprises au cours du film et qui reflète parfaitement l'état d'esprit et le bon
sens de celui-ci.

Mais dès lors qu'il défend un espion russe, Donoan est amalgamé à son client, classé antipatriote. Le peuple américain, conditionné par le prêt-à-pense des médias, plonge dans le déni, refuse d'entendre le

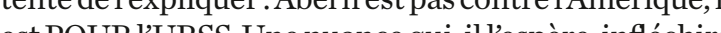
est POURl'URSS. Une nuance qui, ill'espère, infléchira
a sévérité du verdict. st déclaré coupable sur tous les plans par le jury popuest déclare coupable sur tous les plans parleju
laire. Reste au juge à traduire cela en peine.

Donovan qui pressent l'importance stratégique de Donovan qui pressent l'importance stratégique de
on client tente, avant que la peine ne soit prononcée, son client tente, avant que la peine ne soit prononceé,
de convaincre le juge qu'Abel a plus de valeur vivant que mort. L'avenir prouvera qu'il avait vu juste. De mauvaise grâce mais convaincu, le juge condamne Abel à trente ans. Pendant ce temps, des U2 américains s'apprêtent à
décoller pourl'URSS. L'un d'eux est abattu et son pilote, décoller pour l'URSS. L'un d'eux est abattu etso

Forts de cet otage et probablement pour les mêmes raisons que les Américains (crainte que le détenu ne énonce le réseau), les Russes proposent à ceux-ci d'échanger leurs ressortissants respectifs. Cela doit arguments d'un Donovan qui, s'il n'excuse pas le délit,

Le procès a lieu et comme il fallait s'y attendre, Abe se faire secrètement et Donovan est approché pour la tâche. Il accepte, prétexte à sa femme qu'il part pêche

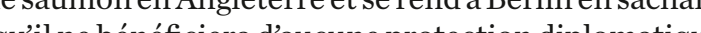
en cas de pépin.

\section{SUSPENSE, TENSION}

La première moitié du film se concentre sur combat mené par Donovan pour éviter la mort à son client en 1957. La seconde le suit à Berlin négocier pour obtenir l'échange de Powers avec Abel. À part qu'entretemps, et de son propre chef, Donovan décide d'exige Pryor. Ce Powers, un étudiant américain, Freder cyor. Ce que la clis a caprice, susceptible de faire capoter la négociation.

Même si on en connait le dénouement, il règne un vrai suspense à la fin du film: les deux clans sont sur le pont, face à face. On attend le signal pour que chaque prisonnier entame sa marche, croise son « challenger et se retrouve parmi les siens. Et ce signal est précisément Frederic Pryor, dont il avait eté convenu qu Checkpos Chane trentane de kilomètres de là, à Berlin entre les zones russe et américaine.

Dans le film, cet étudiant, n’a pas le temps de revenir à l'Ouest, la dernière brique du mur étant placée juste au moment où il allait passer. Etant Américain, cela aurait pu sarranger. Le problème est qu'il portait d'études qui dissertait sur le communisme un traval déte ments suffisants pour qu'il soit Donova lapprect

Donovan lapprend et décide de l'intégrer dans les négociations. Le hiccest que Pryor relevve de l'autorité est-áricande qui veut être considérè aux yeux des nón comme un sous-fifre des Soviétiques. Tout ail de Donovan conste dovì respont Tout le traall de Donovan consistera donc à responsabiliser les Allemands de Estenleur faisant comprendreques refusent, Abel, condan à demeurer en Amérique, pour àt bépondre aux Soviétiques. lors à en répondre aux Soviétiques.

Cette histoire, que n’aurait pas reniée John le Carré Gregory Peck voulut déjà l'adapter au cinéma en 196 climait éte Donovan et Alec Guinness, Abel. Mais le a tension générée par l'affaire des missiles russes insallés à Cuba, le moment était mal choisi.

PARENTHÈSE

Et puisque l'on évoque Cuba, il faut savoir que Donové aurés du succes renporté à Benn, fut

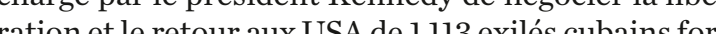
més pura 'est laffaire dite de la Baie des Cochons où ces exilés débt lafuire dite de la Bied. Donovans on ces exilés ieurs fois le Jíder Máximo et, commentu avec Abel, lider Máxino et, conme ce fut le cas de convictions, s'installa tlo àrent jusqu' conus ensemble, Donovan offrant même à Castro une montre que celui-ci sempressa de porter. Le 21 décembre 1962 prision prence pres ne les néritions qui se poursuivirent encore deux ans

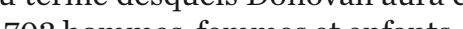

Tout cela pour préciser, cette parenthèse étan fermee, que, depuis 1965, menager linformation pou mordinl Dès lors, éviter d'aborder des tensions amérordial. Dès lors, eviter dabor des destons américano-russes à un moment où la guerre syrienne (par nement plus de mise

\section{DE CHARMAN À SPIELBERG}

À l'origine de Bridge of Spies, il y a Matt Charman, un scénariste anglais dune trentaine dannées. Cest en Unfinished Life. John F Kennedy, 1917-1963 de (A Dallek), qu'il relève, dans le chapitre cons dé a Dallek), qu'il relève, dans le chapitre consacré à l'epilant que James Donovan, l'avo en qui avait négocie lant que James Donovan, lavocat qui avait négociê libération de Gary Powers. Donovan, Charman n'en vait jamais entendu parler. Intrigué Char l'man n'en d'un persons ende qui avait joué un rôle si lanonymat dun personnage qui avait joué un rôle si important, à lire les journux de l'épita consulter des archives, de Donovan. Il en résultat un synopsis qu'il famille An Don Amrprise, il lui fut rép de Steven Spielberg. Á sa bonne à sa plus grande surprise encore, Spielberg himself $l$ lui téléphona, lui demandant de développer le scénario, .. 
•• prêt qu'il était - tant cela l'enthousiasmait - à intercaler sa réalisation dans un planning pourtant déjà bien rempli.

\section{LES FRÈRES COEN À LA RESCOUSSE}

Si malgré sa complexité, le récit est limpide, c'est aussi grâce à l'écriture naturelle et concise des frères Coen (le team qui réalisa, entre autres, The Big Lebowski) qu'on le doit. Ils peaufinèrent la première mouture du scénario écrite par Charman, la rendant plus vivante, moins factuelle, lui imposant une cadence qui compensait le rythme, plus posé que de coutume, de Spielberg. Aucun mot de trop, chaque phrase prononcée est signifiante. Ce sont eux qui ont contacté le réalisateur après avoir eu vent du projet. Il avait déjà été le producteur exécutif de leur remake de True Grit. Ils dépeignent ici l'absurdité de certaines situations, les dédramatisant sans les banaliser lorsqu'elles sont trop tendues, établissant - sous forme de légers traits d'humour - une distance par rapport à elles. Tout est maîtrisé, aucune panique ne transparait dans l'écriture, à l'image de Donovan qui marche sereinement sur les charbons ardents qui pavent sa mission.

Le film totalise 142 minutes durant lesquelles on ne s'ennuie pas une seconde. Hormis la scène où l'avion de Powers est abattu, ce n'est pas le spectacle qui capte, c'est la narration par l'image, une discipline innée chez Spielberg, qui a toujours été son point fort et qui justifie pleinement, à l'instar d'un Capra, d'un Hitchcock, d'un Truffaut ou d'un Lean, son statut de vrai cinéaste.

Quelques petits soucis émaillèrent la sortie du film. Giles Whittell, un ancien correspondant du Times à Moscou réclamant des droits pour le titre qui est aussi celui de son livre (sorti en 2010) lequel traite du même thème (l'échange de prisonniers durant la guerre froide), mais pas sous le même angle. Le film est basé en partie sur le livre écrit en 1964 par James B. Donovan lui-même, Strangers on a Bridge (en français : L'affaire Abel [1965], Paris, L’Archipel, 2015) et centré autour de ce dernier. Alors que dans le livre de Whittell, on est dans le sillage des trois prisonniers.

\section{LE CASTING}

Tom Hanks c'est le James Stewart de notre époque, le voisin que l'on rencontre sur le palier et avec lequel on a tout de suite envie de sympathiser. Qui mieux que lui pouvait incarner James Donovan ? S'il n’a pas les traits d'un Brad Pitt ni le charisme d'un Day Lewis, il dégage suffisamment de personnalité et d'humanité pour que l'on s'y attache et le suive dans ses choix. Aussi Hanks, sans en être la copie conforme, ressemble assez à son modèle. Disons que ce dernier était plus dégarni.

Mais la surprise du film, c'est Mark Rylance : une révélation! Parfaitement inconnu du grand public, il EST Rudolf Abel. Impressionnant par un calme qui déroute, il joue au maximum la sobriété, levant là un sourcil, répondant ici d'un regard, usant d'un ton monocorde lorsqu'il converse. Le genre de prestation qui mérite un Oscar. Qu'il a d'ailleurs obtenu haut les votes !

On relève également Alan Alda, dans le rôle de Thomas Watters Jr, l'associé avec lequel Donovan créa son bureau d'avocats. Alda eut son quart d'heure de gloire dans les années 1970 avec la série télévisée inspirée par $M^{*} A^{*} S^{*} H$.

Côté coulisses, le fidèle chef opérateur Janusz Kaminski continue à se distinguer par un style qui lui est propre : lumières sous-exposées, clair-obscur, couleurs désaturées, tout ce qui a marqué les Spielberg (saufle Tintin en images de synthèse) depuis 1993, année où Kaminski fut le directeur de photo de Schindler's List / La liste de Schindler.

Par contre, John Williams n'a pas écrit la musique. Des ennuis de santé l'en ont empêché. C'est Thomas Newman (cousin de Randy) qui a pris le relais, et ce, de manière plus qu'honorable.

Quel public le film concerne-t-il ? Au niveau générationnel, le mur de Berlin n'est plus, pour la génération rap en tout cas, un référent. Que ce soit ce mur, la Révolution française ou la guerre 14-18, pour elle c'est pareil, cela relève davantage du cours d'Histoire. Dans tous ces cas, elle n'était pas née et ne s'en sent donc pas contemporaine. Reste le génie de Spielberg qui transcende les époques. Et cela devrait suffire à les pousser à voir le film. Et même à le revoir. I

Erik Machielsen

\section{Plus d'infos}

$\rightarrow$ Bridge of Spies / Le Pont des espions de Steven Spielberg (2014).

Avec: Tom Hanks (James B. Donovan), Mark Rylance (Rudolf Abel), Amy Ryan (Mary Donovan), Alan Alda (Thomas Watters Jr.) - 142 minutes. 